\title{
A POSSIBILITY OF DIRECT DETERMINATION OF THE STAR PHASE DENSITY \\ IN THE GALACTIC NUCLEAR CLUSTER
}

\section{A.M. FRIDMAN, E.V. POLYACHENKO AND V.L. POLYACHENKO Institute of Astronomy, Russian Academy of Science, Pyatnitskaya 48, Moscow, 109017 Russia}

Line-of-sight and proper motions data for stars near the Galactic center have evolved in recent years (see, e.g. Eckart \& Genzel). The data were used for determining the star velocity dispersions in different directions (with a result that the velocity distribution is isotropic to within the observational error). Here we note that there exists a possibility for determining the star phase density itself from such data. The method is based on the solution of the corrensponding integral equations with their left sides being calculated from radial velocities and proper motions. Remind that discussion of the problems connected with reconstruction of the phase density from the radial velocity data was started by Merritt. There is some difference in applying this approach to self-gravitating systems (open and globular clusters) and clusters within external gravitational field. Among the latter is, for example, the inner parts of the Galactic nuclear cluster: they are governed by the field of the central black hole. It follows from the proper motions data estimates for a mass within a radius of $0.16 p c$ and within $0.083 p c$ given by Eckart \& Genzel (cited above). In the case of the cluster in the field of the black hole we have no need of information about the densities $\sigma(s)(s$ is the projected radius) and $\rho(r)$ (since the method uses only the gravitational potential $\Phi(r)$, which here is foregone: $\left.\Phi(r)=-G M_{h} / r\right)$. Unfortunately, the data on the stars around the central black hole are yet inadequate to derive the distribution function with satisfactory accuracy. (Obviously the necessary data will be available before long.) So we restrict ourselves to only testing the computer algorithms used on the example of the simplest (Plummer) model, for which $f(E) \propto(-E)^{7 / 2}$.

If a number of stars in the region $s, s+d s ; v_{s}, v_{s}+d v_{s}, v_{\phi}, v_{\phi}+d v_{\phi}$ : $d n=F\left(s, v_{s}, v_{\phi}\right) \cdot 2 \pi s d s d v_{s} d v_{\phi}(F$ should be determined from observations 

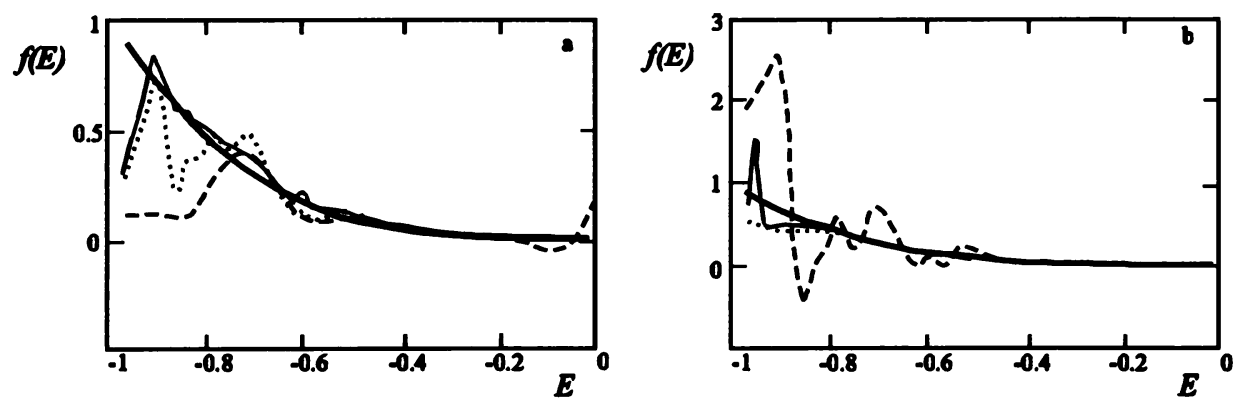

Figure 1. Solutions $f=f^{(s)}(E)$ of the integral equations for approximate left sides (an accuracy is characterized by a value of the quantity $p$ ); a) proper motions, b) line-of-sight velocities: the smoothed solid lines correspond to $f(E)=f_{0}(E) \propto(-E)^{7 / 2}$, the second solid line at each figure shows the solution for $p=10 \%$, the dotted line - for $p=20 \%$, and the dashed line - for $p=30 \%$.

of proper motions), the integral equation for isotropic phase density $f=$ $f(E)$ can be easily derived in the form:

$$
u_{s}(y)=\int_{\phi}^{0} K_{s}(E-y) f(E) d E,
$$

where the kernel $K_{s}(x)=\sqrt{2} \theta(x-\phi) \cdot \int_{0}^{z_{\max }}\left(x-\Phi\left(\sqrt{s^{2}+z^{2}}\right)\right)^{-1 / 2} d z$; $z_{\text {max }}(s, x)$ is the root of the equation $x=\Phi\left(\sqrt{s^{2}+z_{\text {max }}^{2}}\right)$. When lineof-sight velocities are observed, we obtain the same equation (1) with the kernel $K_{s}=\theta(x-\phi) \cdot z_{m a x}^{(s)}(x)$, where $z_{\text {max }}^{(s)}(x)$ is the root of the equation $x=\Phi\left(\sqrt{s^{2}+z^{2}}\right)$.

The Fredholm equations of the form (1) fall into the class of the ill-posed problems; methods of their solution were mainly developed by Tikhonov and his school [see, e.g., Tikhonov et al.]. Fig. 1 presents the solutions for three values of $p \neq 0$, at some fixed value of $s$. These results were obtained with the help of general algorithms without recourse to any $a$ priori information about a form of the distribution function $f(E)$. If such information is available, the results can be sufficiently improved.

\section{References}

Eckart, and Genzel, (1996) Nature 383, p. 415

Merritt, D., (1993) Astrophys. J. 413, p. 79

Tikhonov A.N., Goncharskij A.V., Stepanov V.V. and Yagola A.G. (1990) Numerical methods of solution of ill-posed problems, Moscow, Nauka 